\title{
DEL INFINITO POTENCIAL AL ACTUAL: UN RECORRIDO HISTÓRICO A TRAVÉS DE LA METÁFORA CONCEPTUAL
}

\author{
Tamara Díaz-Chang ${ }^{1,2}$ \\ tamara.diaz@uach.cl \\ https://orcid.org/0000-0001-7349-5057 \\ Elizabeth-H Arredondo ${ }^{1}$ \\ elizabeth.hernandez@ulagos.cl \\ https://orcid.org/0000-0002-5285-1603 \\ Universidad de Los Lagos ${ }^{1}$ \\ Universidad Austral de Chile ${ }^{2}$
}

Recibido: 27 de diciembre de 2020 Aceptado: 19 de mayo de 2021

\section{Resumen}

En este artículo abordamos el análisis histórico y epistemológico del infinito como concepto matemático, mirado bajo el lente de la metáfora conceptual, tratando de precisar los obstáculos que impidieron, por largos períodos de nuestra historia, la aceptación del infinito actual, permitiéndose solamente la existencia del infinito potencial. Argumentamos que este análisis es un tema de especial relevancia a considerar en las agendas de investigación dentro de la didáctica de las matemáticas. En particular, mostraremos cómo el desarrollo de ciertas metáforas conceptuales condujo a un proceso de axiomatización del infinito actual, que concluyó con los trabajos de Georg Cantor. La metodología implementada se apoya en una investigación bibliográfica de carácter cualitativo y argumentativo fundamentada en una metaetnografía. A partir de esta investigación, se obtiene información sobre las estructuras matemáticas que transitan entre los diferentes dominios de partida y de llegada de las metáforas conceptuales, a través de las cuales se desarrolló el infinito matemático durante cuatro estadios principales de la historia, mostrando además la transición del infinito potencial al infinito actual. En particular, se identifican al menos cinco metáforas diferentes que el profesor debe considerar y el estudiante debe desarrollar para lograr una comprensión adecuada del infinito matemático. Palabras clave: Infinito potencial; Infinito actual; Metáfora conceptual.

\section{FROM POTENTIAL TO ACTUAL INFINITY: A HISTORICAL JOURNEY THROUGH CONCEPTUAL METAPHOR}

\begin{abstract}
In this article we address the historical and epistemological analysis of infinity as a mathematical concept, seen through the lens of conceptual metaphors, trying to specify the obstacles that prevented, for long periods of our history, the acceptance of actual infinity, allowing only the existence of potential infinite. We argue that this kind of analysis is of special relevance to be considered in research agendas within the didactics of mathematics. In particular, we will show how the development of certain conceptual metaphors led to a process of axiomatization of actual infinity, which ended with the works of Georg Cantor. The implemented methodology is supported by a qualitative and argumentative bibliographic research based on a metaethnography. From this research, information is obtained about the mathematical structures
\end{abstract}


which are been mapped between starting and ending domains of conceptual metaphors, through which mathematical infinity was developed during four main stages of history, showing the transition from potential infinity to actual infinity. In particular, at least five different metaphors are identified that the teacher must consider and the student must develop to achieve a proper understanding of mathematical infinity.

Keywords: Potential infinity; Actual infinity; Conceptual metaphor.

\section{DO POTENCIAL INFINITO AO ATUAL: UMA VIAGEM HISTÓRICA ATRAVÉS DA METÁFORA CONCEITUAL}

\section{Resumo}

Neste artigo abordamos a análise histórica e epistemológica do infinito como conceito matemático, visto pelas lentes da metáfora conceitual, tentando especificar os obstáculos que impediram, por longos períodos de nossa história, a aceitação da existência do infinito atual, permitindo apenas a existência do potencial infinito. Argumentamos que esta análise é um tema de especial relevância a ser considerado nas agendas de pesquisa no âmbito da didática da matemática. Em particular, mostraremos como o desenvolvimento de certas metáforas conceituais levou a um processo de axiomatização do infinito atual, que culminou nas obras de Georg Cantor. A metodologia implementada é sustentada por uma pesquisa bibliográfica qualitativa e argumentativa com base em uma metaetnografia. A partir dessa pesquisa, são obtidas informações sobre as estruturas matemáticas que passam entre os diferentes domínios inicial e final das metáforas conceituais, por meio das quais o infinito matemático foi desenvolvido durante quatro etapas principais da história, mostrando também a transição do infinito potencial para o infinito real. Em particular, são identificadas pelo menos cinco metáforas diferentes que o professor deve considerar e o aluno deve desenvolver para alcançar uma compreensão adequada do infinito matemático.

Palavras-chave: Potencial infinito; Atual infinito; metáfora conceitual.

\section{Introducción}

El infinito matemático es uno de los conceptos más complejos a los que se enfrentan estudiantes y profesores en el aprendizaje de las matemáticas, y por ello ha sido ampliamente estudiado desde diferentes perspectivas teóricas en didáctica de la matemática. A pesar de esto, no es posible afirmar que comprendemos los complicados procesos cognitivos que se desarrollan en relación a su aprendizaje. Existen numerosos trabajos (e.g. Arrigo y D’Amore, 1999; Fischbein, 2001; Tall, 1991) que dan cuenta de la dificultad y complejidad que presenta, en este caso, el obstáculo epistemológico. En particular, Arrigo y D’Amore (2004) plantean que el aprendizaje de la concepción actual del infinito matemático se produce lentamente, de modo contradictorio, tras un largo proceso de maduración y sistematización cognitiva. 
De manera general, existen diferentes teorías en la investigación en didáctica de las matemáticas que intentan dar respuesta a la pregunta esencial: ¿Cómo se aprende en matemáticas? Algunas de estas teorías sostienen que creamos nuestros sistemas conceptuales con la ayuda de metáforas, que establecen correspondencias que solo pueden surgir a través del uso del lenguaje, y que indican el carácter social y supraindividual del aprendizaje. Dentro de este contexto también se afirma que la proyección metafórica es un mecanismo recursivo a través del cual la cultura se perpetúa y se reproduce en un sistema de conceptos que crece continuamente (Sfard, 2008). Generalmente, se reconoce que en nuestra vida diaria el uso de metáforas nos ayuda a comunicarnos de manera efectiva, a comprender tópicos complejos, a la vez que condiciona nuestras actitudes y nuestros razonamientos (Flusberg, Matlock y Thibodeau, 2018). De manera más específica, las denominadas metáforas conceptuales aparecen en los estudios de lingüística cognitiva que se apoyan en la teoría de la Embodiment Cognition (EC) (Rosch, Thompson, y Varela, 1991). Esta teoría considera que ciertos sistemas conceptuales humanos se basan en simulaciones sensomotoras de modalidades específicas del funcionamiento del cerebro humano, y que el cerebro, el cuerpo y el medio ambiente del ser humano están acoplados dinámicamente y se influyen entre sí. Apoyándose en esta teoría cognitiva Lakoff y Núñez (2000) proponen que el aprendizaje de la concepción actual del infinito matemático se basa en un tipo de metáfora conceptual, mediante la cual, los procesos que continúan indefinidamente se conceptualizan de manera que alcanzan un resultado final, una especie de "completamiento metafórico".

Notemos que asumir como válida esta teoría cognitiva también tiene implicaciones sobre las creencias epistemológicas básicas sobre las que se construye el discurso del profesor de matemáticas. En la actualidad existen dos acercamientos radicalmente opuestos hacia el aprendizaje que guían dicho discurso, y que constituyen en sí mismas, metáforas: la metáfora de la adquisición y la metáfora de la participación (Sfard, 2008). La metáfora de la adquisición es la que subyace a nuestra idea sobre el aprendizaje en general. En este caso aprender se entiende como ganar posesión de, o adquirir conocimientos, y el estudiante es un sujeto que desarrolla conceptos, que construye conocimientos. En la metáfora de la participación el estudiante es un sujeto interesado en la participación activa en cierta clase de actividades o prácticas matemáticas, y el aprendizaje se ve como la integración del estudiante a una comunidad matemática en acción, lo que implica la habilidad de comunicarse en el lenguaje de 
la comunidad y de actuar según sus normas particulares. Esta nueva visión del aprendizaje propuesta por esta última metáfora, presupone un cambio en el discurso del profesor que lo libera de la paradoja del aprendizaje planteada por Platón en su Menón (Cobb, Yockel y Wood, 1992), mediante la "desobjetivación del conocimiento" (Sfard, 2008). Sin embargo, si consideramos que lo perceptual es origen del pensamiento, entonces no se puede escapar de la objetivación del conocimiento, y por lo tanto, el uso de estas metáforas en el discurso del profesor constituye también fuente de dificultades a las que se enfrenta un estudiante durante el aprendizaje de cualquier concepto matemático.

Por otra parte, para comprender con mayor profundidad los procesos de aprendizaje de un determinado objeto o concepto, y en nuestro caso, del infinito matemático, es importante entender cómo éste fue surgiendo a lo largo de la historia. Esto implica observar cuáles fueron las etapas de mayor o menor desarrollo en las que se puede marcar algún progreso en su conceptualización como ente matemático o de las distintas partes que lo constituyen (Font, 2007). Para abordar este tipo de análisis histórico del infinito como concepto matemático desde una perspectiva epistemológica es útil el estudio de los mecanismos metafóricos, y en especial, de las metáforas conceptuales que condujeron a su proceso de axiomatización, proceso que culminó en el siglo XIX con los trabajos de Georg Cantor. Es precisamente en el estudio de estas metáforas conceptuales y en su surgimiento a lo largo de la historia, en el que nos centraremos en este trabajo, con el objetivo de identificar dificultades, que en relación a estas metáforas, el estudiante debe vencer durante el aprendizaje de este concepto matemático.

\section{Marco Teórico}

En el estudio de un concepto desde el punto de vista histórico y epistemológico se analiza no solamente la constitución y función técnica de dicho concepto, sino también la manera compleja en que los desarrollos teóricos se producen, para llegar a convertirse en elementos de discursos matemáticos con significados determinados y reconocimiento en una época dada.

Siguiendo esta premisa de investigación, para fundamentar nuestro análisis nos apoyaremos en los estudios de la lingüística cognitiva realizados por Lakoff y Núñez (2000), basados en la teoría de la EC (Rosch, Thompson, y Varela, 1991), que propone que ciertos procesos constituyentes de la cognición están basados y se derivan de la interacción del medio con el individuo, y que esta interacción genera que conceptos abstractos como los matemáticos 
puedan emerger a través de sus percepciones y acciones corporales por medio de images schemas (Rohrer, 2006). A pesar de que nadie sostiene que la $E C$ sea el único fundamento sobre el cual se basa el pensamiento y el lenguaje humanos, los estudios experimentales que se han realizado cada vez con mayor frecuencia en los últimos años, indican que este tipo de mecanismo a menudo juega un papel muy importante en la forma en que los seres humanos le damos sentido a nuestro mundo (Wilson y Gibbs, 2007).

Además, la lingüística cognitiva nos ofrece un conjunto de técnicas para estudiar estructuras conceptuales implícitas en nuestras experiencias y que son, en gran parte, inconscientes, jugando un rol fundamental en la construcción de ideas matemáticas (Gallese y Lakoff, 2005; Lakoff y Núñez, 2000). Desde esta perspectiva, images schemas pueden derivar en mecanismos cognitivos conocidos como metáforas conceptuales, que constituyen asociaciones o enlaces inconscientes entre dominios conceptuales diferentes, en el que la estructura del dominio de partida infiere una estructura en el dominio de llegada. El origen de una metáfora conceptual puede ser una experiencia del mundo físico, así como una conceptualización ya existente, creando un vínculo que conecta conceptos o subdominios, fuera o dentro de las matemáticas, como se muestra en la Figura 1.

Otro de los mecanismos cognitivos relevantes en este tipo de análisis es la integración conceptual, que también constituye una asociación o enlace inconsciente entre dominios conceptuales diferentes, pero a diferencia de la metáfora conceptual que representa una aplicación desde un único dominio conceptual de partida hacia un dominio conceptual de llegada, en este caso se parte de varios dominios que se conectan para "proyectarse" selectivamente en un dominio de llegada donde se desarrolla una estructura nueva.

Figura 1. La metáfora conceptual

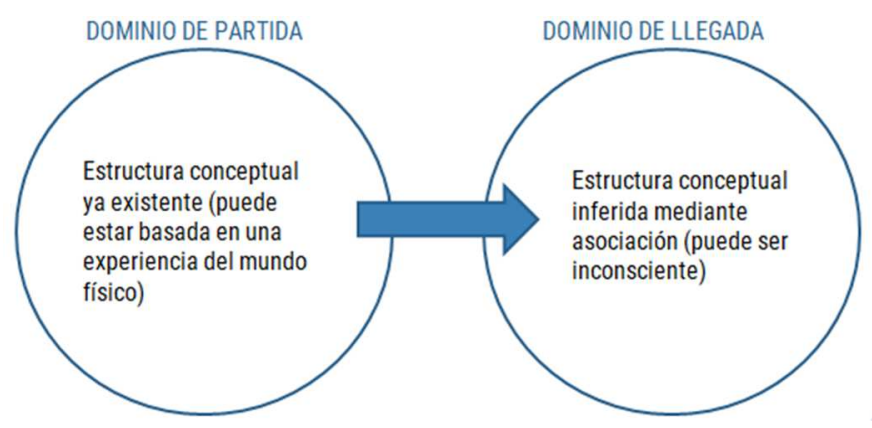

Fuente: Elaboración propia 
Para entender la naturaleza cognitiva y la estructura conceptual subyacente al infinito matemático, nos referiremos a dos dimensiones principales de los fenómenos cognitivos que ocurren en este caso: uno es el aspecto (Comrie, 1976), y el otro es la metáfora básica del infinito (MBI) (Lakoff y Núñez, 2000). Tanto el aspecto como la MBI, son mecanismos cognitivos que se basan en la $E C$.

Este marco teórico nos permitirá mostrar el desarrollo y la evolución de algunas de las metáforas conceptuales que caracterizan la construcción del infinito matemático como concepto clave y soporte fundamental del sólido edificio matemático a lo largo de la historia. Partimos de la premisa de que la naturaleza de las matemáticas se puede apreciar de forma más clara a partir de estas metáforas conceptuales, y especialmente a partir de las metáforas que se vinculan con el infinito matemático, ya que éste se encuentra en el centro de numerosos dominios fundamentales como la geometría proyectiva, el cálculo infinitesimal, la topología o la teoría de conjuntos, por solo citar algunos.

\section{Marco Metodológico}

Como ya hemos mencionado, el objetivo de esta investigación es identificar las dificultades, que en relación a las metáforas conceptuales, el estudiante debe enfrentar durante el aprendizaje de este concepto matemático. Para lograr este objetivo nos proponemos obtener información sobre los aspectos y las estructuras matemáticas que transitan entre los diferentes dominios de partida y de llegada de las metáforas conceptuales, a través de las cuales se desarrolló el proceso de axiomatización del infinito matemático durante distintas etapas de la historia, mostrando además la transición del infinito potencial al infinito actual. En particular, se trata de identificar las diferentes metáforas conceptuales que el estudiante debe desarrollar para lograr una comprensión adecuada del infinito matemático.

La metodología implementada se apoya en la investigación bibliográfica de carácter cualitativo y argumentativo. Para realizar la selección de la literatura, y la interpretación y el análisis de la información extraída, se utiliza el método de la meta-etnografía (Noblit y Hare, 1998) que nos permite sintetizar de manera sistemática los resultados de nuestra investigación y dar respuesta focalizada a las preguntas establecidas por el objetivo de investigación mencionado anteriormente. Este método nos proporciona una forma específica de realizar metasíntesis cuya meta va más allá del resumen y el análisis de datos, donde los hallazgos de los 
estudios originales se convierten en datos que son analizados a través de un proceso riguroso de interpretación y comparación de ideas, conceptos y perspectivas relevantes, conduciendo así a la síntesis esperada.

En la meta-etnografía los estudios pueden relacionarse entre sí de tres maneras: primero, estos pueden ser directamente comparables como traslaciones recíprocas, es decir, que con los conceptos usados se busca el grupo de ideas dominantes que pueden abarcar otros nuevos grupos de conceptos o metáforas. Segundo, ellos pueden estar en oposición o como traslaciones que se refutan. Y tercero, los estudios en conjunto pueden presentar una línea de argumentos.

Para llevar a cabo nuestra investigación seleccionamos noventa documentos entre libros de historia de las matemáticas y de matemáticas avanzadas, artículos históricos y ediciones de manuales originales, publicados en el período comprendido entre los años 1985 y 2020 , y donde se tratan temas relacionados con el desarrollo del infinito matemático.

El método fue implementado siguiendo las siete etapas propuestas por Noblit y Hare: 1) se inició y decidió el fenómeno a estudiar, identificando áreas de interés útiles para la síntesis; 2) se decidió qué estudios cualitativos eran relevantes, lo cual requirió determinar el alcance de la síntesis propuesta; 3) se realizó la lectura crítica de los estudios seleccionados para identificar los conceptos principales y la extracción de las metáforas interpretativas; 4) se determinó la relación entre los estudios: esta etapa incluyó la elaboración de listas de metáforas claves en cada estudio y sus relaciones con otros estudios; 5) se realizaron las traslaciones entre los estudios, es decir, se tomaron los conceptos intactos de cada estudio para permitir la comparación con los otros; 6) se hizo una síntesis de las traslaciones, llegando a una nueva interpretación de la teoría; y por último en la fase final correspondiente al paso 7), la síntesis ha sido expresada.

\section{Análisis y resultados}

En esta sección presentaremos primeramente un análisis de la evolución del infinito como concepto matemático a lo largo de la historia, centrándonos en cuatro períodos principales de su desarrollo. Posteriormente analizaremos dichos períodos históricos reconociendo ideas y paradojas contra-intuitivas que surgieron en relación a éste, desde la perspectiva de la $E C$ y la lingüística cognitiva, identificando así las metáforas conceptuales que se originaron en su 
proceso de axiomatización y que los estudiantes deben desarrollar para lograr una comprensión adecuada de dicho concepto.

\section{Análisis de la evolución del infinito matemático a través de períodos históricos}

A continuación analizaremos la evolución histórica del infinito matemático, con el propósito de identificar las metáforas conceptuales que se originaron a lo largo de la historia, permitiéndonos crear este concepto matemático. A partir de la investigación realizada, nuestro análisis se centrará en cuatro períodos históricos principales que son relevantes para estudiar el desarrollo del infinito matemático desde nuestra perspectiva.

Comenzaremos nuestro análisis destacando que, desde la más remota antigüedad, los hombres se fascinaron con el infinito. Este es, indiscutiblemente, uno de los conceptos más intrigantes y controversiales que haya creado la mente humana, y por lo general, las nociones que tenemos en relación a éste, se originan como extrapolación de nuestra existencia física, limitada y finita. A lo largo de la historia hubo prolongados debates acerca de su naturaleza.

\section{La Grecia Antigua (Siglos VI - III a. C.)}

Ya desde el siglo VI a. C. los filósofos Pre-socráticos se interesaron por especulaciones acerca del infinito. Anaximandro fue uno de los primeros en considerarlo rigurosamente para responder a preguntas acerca del origen, la naturaleza y los límites del universo (Stillwell, 2010), como el apeiron, algo neutral, imperecedero, sin final, ilimitado, asociado a Dios (Guthrie, 2000). Hacia el siglo IV a. C. algunos atomistas, como Leucipo y su discípulo Demócrito, pensaban que la materia estaba compuesta por un número infinito de "indivisibles" (Lucrecio, trad. 1985), y afirmaban además que el universo era infinito, sin embargo Parménides y su discípulo Zenón estaban en desacuerdo.

Por esa época existían concepciones opuestas sobre el espacio y el tiempo, y en consecuencia, en la Grecia antigua se concebía al infinito de dos formas diferentes: el infinito potencial y el infinito actual. Específicamente, estas dos concepciones del infinito como objeto matemático tienen su origen en las categorías filosóficas definidas por Aristóteles en su Metafísica (Aristóteles, trad. 1985), cuando distingue entre el ser "en potencia” y el ser "en acto", refiriéndose al apeiron de Anaximandro desde una perspectiva diferente. Considerando una colección de números, argumentaba que la "totalidad" de los números no podía estar presente en nuestro razonamiento, puesto que al generar una lista de éstos, uno por uno, no se 
podía generar la lista completa. Es decir, siempre existiría un número que no había sido considerado antes. Por tanto, argumentaba que el apeiron no era algo "agotado" fuera de lo cual no hay nada, sino algo fuera de lo cual siempre hay algo, y en consecuencia, era "inagotable". Así es que el apeiron no podía ser visto como una totalidad completa, porque lo que es completo tiene fin, tiene un elemento límite, luego el apeiron estaba caracterizado por su inherente incompletitud, por su existencia solo como potencialidad. Por lo que el infinito tenía solo una “existencia potencial", no como algo real manifestado.

Desde el punto de vista matemático, la noción de infinito potencial se centra en la operación reiterativa e ilimitada, es decir, en procesos recursivos interminables. Esta noción de infinito también se refiere a lo infinitamente pequeño o a lo infinitamente grande, las iteraciones hasta el infinito en límites continuos o en fractales. El infinito potencial fue considerado en la filosofía eleática del siglo $\mathrm{V}$ a. C. en el estudio de sucesiones y sumas de series, a través de las paradojas de Zenón, que intentaban mostrar que distintas concepciones sobre el infinito llevaban a situaciones en apariencia contradictorias. Ejemplo de esto lo son las conocidas paradojas de Aquiles y la tortuga, la del movimiento; o la de la dicotomía, donde el tiempo y el espacio son magnitudes infinitamente divisibles (Bolzano, 1991).

Por otra parte, el infinito actual se refiere a un infinito existente como un todo o unidad, como el resultado final de un proceso. Esta noción de infinito apareció también en la matemática desarrollada por otras civilizaciones antiguas de China y la India. Por ejemplo, para los matemáticos hindúes del siglo III a.C., el infinito estaba representado por una cantidad que no cambiaba sin importar cuántos números se le adicionasen o se le substrajesen (Paéz, 2013).

Una de las características más interesantes de la matemática de la escuela Pitagórica fue el rechazo que le hicieron a sus inconmensurables, como parte del rechazo general que sentían por los procesos infinitos. El desarrollo posterior de las matemáticas en la Grecia del siglo IV a. C. se caracterizó por la influencia de dos escuelas filosóficas fundadas en Atenas, la Academia fundada por Platón, y el Liceo creado por Aristóteles, donde se enseñaban sus ideas, que ejercerían gran influencia en las matemáticas griegas de los siglos posteriores. Un ejemplo relevante de esta influencia, es el hecho de que Arquímedes estuvo muy cerca del moderno concepto de infinito matemático hacia el siglo III a. C., a pesar de que en sus obras solo menciona al infinito dos veces en El Arenario (Arquímedes, trad. 1987). De hecho, se tiene 
evidencia de que no solo trató con el infinito potencial, sino que también consideraba que no todos los objetos infinitos en número eran iguales (O’Connor \& Robertson, 2002). En El Método, se muestra que investigó el número infinito de objetos considerando tres pares de magnitudes infinitas, argumentando que eran iguales en número respectivamente. Esto sugiere que él consideraba que no todos los conjuntos infinitos de objetos eran iguales en número, y que tenía nociones acerca del concepto de cardinal que posteriormente precisó Cantor, y que aceptaba la existencia del infinito actual, y de infinitos más grandes que otros.

También en El Método, Arquímedes revelaba cómo hallaba sus resultados usando argumentos relacionados con el infinito, para luego probarlos rigurosamente omitiendo dichos argumentos. Para él, el infinito podía ser usado para descubrir resultados que no eran inicialmente accesibles por medio de argumentos lógicos. Siguiendo esta idea, por ejemplo, en sus trabajos también consideró que la suma de una serie infinita podía ser finita (Bagni, 2004).

Los antiguos griegos no tuvieron el valor de aceptar la existencia del infinito de manera abierta, al principio a causa de las paradojas de Zenón (que producían contradicciones lógicas, tanto si se consideraba el espacio y el tiempo como continuos, que como discontinuos), y más tarde debido a la influencia de las ideas de Aristóteles. Se puede decir que fueron muy cuidadosos de evitar al infinito como el resultado final de cualquier proceso iterativo (como el infinito que emerge en el paso al límite) y en general, al infinito actual, en sus argumentos y demostraciones. En consecuencia, durante siglos los matemáticos se debatieron en contra de la existencia de este infinito, aceptando solamente al infinito potencial (Bussotti, 2014).

\section{El Medioevo y el Renacimiento (Siglos XIV-XVII)}

En el siglo XIV el infinito reapareció en la India, en los trabajos de Madhava y la escuela de Kerala. En estos trabajos se desarrollaron ideas fundamentales como el paso al límite en la expansión series de Taylor de funciones trigonométricas y de otras series de potencias, así como la aproximación racional de series infinitas. También se desarrolló, antes que en las matemáticas europeas, el trabajo con fracciones continuas infinitas (Paéz, 2013).

Por esta misma época, en el pre-renacimiento europeo, a partir de los trabajos de Arquímedes, se progresó en el desarrollo de técnicas infinitesimales para calcular áreas y volúmenes. En esta primera etapa se introdujeron los conceptos de indivisibles e infinitésimos, como el germen para lo que luego serían los infinitesimales. Entre los primeros trabajos que 
destacaron, se pueden citar los indivisibles de Galileo, Kepler y Cavalieri. En su obra Discorsi e dimostrazioni matimatiche (1638), Galileo argumenta que el continuo está formado por indivisibles cuando discute el tema del infinito, partiendo de la misma idea aristotélica: la indivisibilidad indefinida, pero partiendo de un concepto opuesto al de Aristóteles, que lo concebía como un proceso sin final. Para Galileo el infinito actual era algo terminado. A partir de sus indivisibles presentó ciertas objeciones respecto al infinito mediante el uso de paradojas. Por ejemplo, a partir de la famosa paradoja que lleva su nombre, señaló que si el número de números naturales no era solo potencialmente infinito, sino también actualmente infinito, entonces existían tantos números cuadrados perfectos como números naturales, puesto que ambos conjuntos podían ponerse en correspondencia biyectiva. Sin embargo, argumentaba, sobre la base del axioma de Euclides: "el todo es mayor que cualquiera de sus partes”, que debía existir una cantidad mayor de números naturales que de números cuadrados perfectos. Al llegar a esta contradicción, concluyó que las cantidades infinitas eran incomparables. Galileo fue uno de los primeros en notar que: "Las dificultades aparecen cuando intentamos, con nuestras mentes finitas, discutir el infinito, asignándole a éste propiedades que le damos a lo finito y limitado". (Galileo, 1954/1638, p.31)

En la Italia del siglo XV, Nicolás de Cusa, inspirado en los trabajos de Euclides y Arquímedes, consideraba que un círculo era un polígono con un número infinito de lados, por lo que implícitamente estaba asumiendo al infinito actual como el resultado final de un proceso. Esto a su vez inspiró a Kepler en el siglo XVII, a escribir en su Opera Omnia que no existía diferencia esencial entre un polígono y un círculo, un círculo y una elipse, un área infinitesimal y una recta, y por tanto, entre lo finito y lo infinito, lo cual resultaba contradictorio (Tall, 1991). En su obra Nova Stereometria Doliorum Vinariorum de 1615, siguiendo los pasos de Arquímedes, desarrolló de forma sistemática el cálculo de áreas y volúmenes. El método consistía en seccionar un sólido en un número finito de secciones infinitesimales o sólidos indivisibles, de formas y tamaños convenientes de acuerdo con cada problema en particular. La suma de todos esos indivisibles permitía obtener el volumen buscado. En este trabajo Kepler realizó el paso al límite, identificando una curva con la suma de rectas infinitamente cortas, y un área con la suma de rectángulos infinitamente numerosos e infinitamente pequeños, en vez de usar las demostraciones por reducción al absurdo hechas antes por Arquímedes. 
Siguiendo las ideas de Kepler y Galileo, Cavalieri creó la técnica geométrica conocida como el método de los indivisibles para el cálculo de cuadraturas, que sirvió de base a los métodos infinitesimales que surgirían posteriormente (Bell, 2003). En 1635, en su obra Geometria Indivisibilibus presentó el cálculo del área de una figura plana considerando que estaba formada por un número infinito de segmentos paralelos equidistantes, cada uno de los cuales se interpretaba como un rectángulo infinitamente delgado. Es decir, presentaba al área como la suma de infinitos segmentos rectos (a los que llamaba los indivisibles), y los consideraba como entes de dimensión menor respecto del continuo del cual formaban parte, afirmando que una línea estaba hecha de puntos, un plano de líneas, y un sólido de áreas planas. Su método recurría en principio al infinito, aunque finalmente lo eliminaba, con la ventaja de que estaba basado en una representación del continuo geométricamente intuitiva, eliminando el paso al límite y conservando todas las ventajas de los métodos infinitesimales de Kepler (Bussotti, 2014).

\section{El desarrollo del Cálculo (Siglos XVII - XVIII)}

Otro de los matemáticos que intervino en la crisis producida por el rechazo del infinito, fue Pascal en el siglo XVII, que comprendió que en el método de los indivisibles de Cavalieri aparecía implícitamente lo infinitamente pequeño, por lo que retomó el concepto de un infinito “tan pequeño como se quiera” de Eudoxo. Sin embargo, los indivisibles de Pascal no cumplían algunas reglas de la aritmética. Al asumir que el indivisible era el cero de la aritmética, se oponía al hecho de que un plano estaba compuesto por un número indefinido de líneas. Así es que su noción de indivisible reflejaba la contradicción que había en su método, al considerar la división infinita como algo que no se puede representar ni comprender, y que sin embargo, es un principio geométrico que no se puede eludir. Aún así, mediante su trabajo introdujo los principios básicos del análisis infinitesimal, y a pesar de no manejar de forma explícita el concepto de infinito, comprendió que éste era la clave para el desarrollo de la teoría. Usando su método, pudo calcular límites de sumas de un número infinitamente grande de cantidades infinitamente pequeñas (Bagni, 2006).

A mediados del siglo XVII, el símbolo actual de infinito $(\infty)$ fue introducido por John Wallis, en su obra Arithmetica Infinitorum de 1655. Además consideró el recíproco $(1: \infty)$ que utilizaba para "la nada", e introdujo sistemáticamente el análisis de series infinitas. Por esta 
misma época Fermat introdujo el método de descenso infinito para hacer demostraciones por reducción al absurdo como los griegos, basado en el hecho que, si una proposición es verdadera para algún valor entero positivo $n$, entonces será verdadera para valores enteros positivos menores que $n$. Al no existir una secuencia de descenso infinita en los enteros positivos, este tipo de demostración conduce a una contradicción. Fermat también utilizó la suma de la serie geométrica convergente para calcular cuadraturas, mediante una especie de método de exhaución, y su técnica consistió en considerar rectángulos infinitesimales inscritos en la figura a cuadrar, cuyas bases tenían dimensiones que estaban en progresión geométrica. Su método consideraba la división del área bajo una curva en elementos de área infinitamente pequeños, la aproximación de la suma de esos elementos de área por la suma de rectángulos infinitesimales, y algo similar al límite cuando el número de elementos crecía indefinidamente, mientras que el área de éstos se hacía infinitamente pequeña (Bagni, 2006).

Newton y Leibniz se basaron en estas ideas para desarrollar el Cálculo infinitesimal años después, realizando un gran avance con relación a la formalización del concepto de infinito. Establecieron que el Cálculo se ocupaba de manipular infinitudes, y comprendieron que este cálculo de infinitos se refería a números infinitamente grandes e infinitamente pequeños. Aunque las técnicas desarrolladas por ellos eran más rigurosas que las anteriores, aún presentaban contradicciones en sus fundamentos. En particular, ambos usaban cantidades infinitamente pequeñas que involucraban al infinito actual, tratando de hacerlas compatibles con el infinito potencial de Aristóteles. Newton lo hizo a través de sus últimas y primeras razones, y Leibniz a través de la noción de triángulo característico (Bagni, 2006).

De hecho, en su última obra Quadrature of Curves (1676), Newton expresó su propósito de abandonar el uso de las cantidades infinitesimales, y enunció su teoría de las "razones primera y última de cantidades evanescentes", anticipándose al concepto matemático de límite (las razones últimas por las que las cantidades evanescentes desaparecen son límites a los que tienden a acercarse las razones de cantidades continuamente decrecientes, límites que no pueden traspasarse, ni alcanzarse, antes de que estas cantidades disminuyan in infinitum).

Por su parte, lo novedoso del Cálculo infinitesimal de Leibniz, estaba en la investigación de lo infinito a partir de las diferencias de diversos órdenes de infinitos, considerando lo infinitamente pequeño como variación, lo que le fue sugerido por el tipo de funciones a las que 
le calculó su derivada. Sin embargo, pensaba que los números infinitos debían rechazarse. Para él, el concepto del infinito era lógicamente un absurdo, y si no había números infinitamente grandes, tampoco existían los números infinitamente pequeños.

Posteriormente, Euler publicó un libro de texto donde se hacía una introducción al Cálculo con los infinitos y se estudiaban funciones elementales desarrolladas en series infinitas. Por primera vez se desarrollaron funciones en productos infinitos, y para obtener estos desarrollos, Euler utilizó como herramienta cantidades infinitamente grandes y cantidades infinitamente pequeñas (Durán, 2003).

Hay que destacar que en esta época, con el fin de llegar a acuerdos y hacer avanzar la teoría, los matemáticos recurrían a la intuición, y consideraban como válido en lo infinito, lo que era válido para lo finito. Para ello se empleaban generalmente consideraciones de la geometría euclidiana y de la mecánica. Además, el problema del infinito ya había aparecido en el tratamiento de problemas físicos como el de la cuerda vibrante y la conducción del calor, por lo que se hizo cada vez más indispensable la formalización de este concepto para el desarrollo de las nacientes disciplinas matemáticas como las ecuaciones diferenciales.

\section{La Época Moderna (Siglos XIX-XX)}

En el siglo XIX aparecieron problemas de investigación relacionados con la falta de rigor formal, entre los que se encontraba el tratamiento de los infinitesimales. Cauchy fue el primero en notar la necesidad de considerar este problema conceptual, y comprendió que para construir una base sólida sobre la cual soportar las estructuras matemáticas, primero tenía que establecer una definición clara y precisa de los conceptos de función y de límite. En 1821 en su Analyse algébrique, definió este último, a través del cual incorporó las cantidades infinitamente pequeñas e infinitamente grandes, dejando de considerar así a los infinitésimos con valor fijo, no nulo e indeterminado, como se había hecho anteriormente. En este trabajo definió sus infinitamente pequeños como cantidades variables que tenían como límite al cero. Sin embargo, para él el infinito era el límite de "valores numéricos de una misma variable que crecen más y más de modo que rebasan cualquier número dado", y por tanto no escapaba del enfoque potencial. Respecto al continuo, definió la "variación continua" refiriéndose a "los valores que va tomando sucesivamente una variable". Esto, unido al uso que hacía de los infinitesimales, mostraba dos teorías igualmente válidas del continuo lineal, o sea, las dos concepciones griegas 
de la recta: concebir o no, a la recta formada de puntos. A partir de sus trabajos, Weierstrass estableció la definición formal del límite en el lenguaje épsilon-delta, formalizando así al infinitesimal potencial, años más tarde.

La problemática sobre el infinito potencial e infinito actual se pudo comprender mejor a través de los trabajos de Kant de 1790 (Sanhueza, 2015). Al igual que sus colegas griegos, más allá de las doctrinas Aristotélicas, Kant y los matemáticos de esta época se oponían a aceptar la existencia del infinito actual, debido a las numerosas paradojas y dificultades que éste originaba. A partir de sus trabajos, Bolzano abordó de manera formal y sistemática el problema del infinito actual en su libro Paradojas del infinito (Bolzano, 1991), y su reflexión no se limitó a especulaciones filosóficas o metamatemáticas, sino que también exploró conceptualmente las propiedades de los conjuntos infinitos y abordó la comparación de conjuntos infinitos, retomando las ideas de Galileo, que ya había establecido biyecciones entre conjuntos infinitos y subconjuntos propios de estos en el siglo XVII. Dedekind, siguiendo en la misma dirección de Bolzano, comprendió que debía caracterizar rigurosamente los números irracionales a través del concepto de cortadura, y partiendo del hecho que el continuo lineal no se "llenaba" completamente con los racionales. A partir de esto, y después de muchos siglos de contradicciones, Dedekind logró una definición precisa de conjuntos infinitos (Eves y Carroll, 1966). Esta definición, que finalmente cuestionaba la propiedad de los conjuntos finitos que afirma que "el todo es mayor que la parte", se basó en el concepto de potencia y se enunciaba así: un conjunto $A$ es infinito si existe un subconjunto propio $B$ de $A$ tal que $B$ tiene la misma potencia de $A$.

Sin embargo, fueron los trabajos de Georg Cantor en 1883 los que, desarrollando las ideas de Bolzano y Dedekind, consagraron el triunfo del infinito actual en las matemáticas modernas. Declaró que siempre que dos conjuntos -finitos o infinitos- pudiesen ser emparejados por una correspondencia biyectiva, entonces tendrían el mismo número de elementos (Jech, 2011). Debido a que existía tal correspondencia entre números naturales y pares, concluyó que había tantos números pares como números naturales. Siguiendo la definición de Dedekind, esto simplemente mostraba una propiedad de los conjuntos infinitos. ¿Y qué había de otros tipos de conjuntos infinitos? ¿Podrían ponerse en correspondencia uno a uno con los números naturales? Y los números naturales y los números pares, ¿se podían ordenar de acuerdo a la magnitud, de 
tal manera que cada miembro tuviese un sucesor definido? Además, ¿qué sucedía, con los números racionales, que no tenían esta propiedad?

Su teoría y su tratamiento de los conjuntos infinitos le condujo a conclusiones incluso más inesperadas, como por ejemplo: la cantidad de puntos en un segmento de recta es mayor que la cantidad de números naturales. Para demostrar lo anterior y que en consecuencia, existía mayor cantidad de números reales que números naturales, utilizó el ingenioso argumento de la diagonal en su demostración por reducción al absurdo. Supuso que el conjunto de todos los números reales comprendidos en un intervalo $(c, d)$ de la recta real, por ejemplo, los números mayores que 0 y menores que 1 , se puede poner en correspondencia biyectiva con el conjunto de los números naturales. Cantor propone construir el conjunto de los elementos del intervalo $(0,1)$ como el conjunto de los elementos de la forma $a_{i}=0, a_{i 1} a_{i 2} a_{i 3} \ldots$ para $i=1,2,3, \ldots, \mathrm{y}$ ordena los elementos de este conjunto de la manera siguiente:

$$
\begin{aligned}
& 1 \rightarrow 0 . a_{11} a_{12} a_{13} \ldots \\
& 2 \rightarrow 0 . a_{21} a_{22} a_{33} \ldots \\
& 3 \rightarrow 0 . a_{31} a_{32} a_{33} \ldots \\
& \ldots \rightarrow \ldots \ldots
\end{aligned}
$$

Sea $b=0, b_{1} b_{2} b_{3} \ldots$ un número real que pertenece al intervalo $(0,1)$, y para el cual cada cifra $i$-ésima de la parte decimal $b_{i}$, es un dígito diferente de $a_{i i}$. Obviamente, el número $b$ es diferente de $a_{1}$ al menos en su primer decimal, o sea $b_{1} \neq a_{11}$, diferente de $a_{2}$ al menos en su segundo decimal, o sea $b_{2} \neq a_{22}$, y en general, diferente de $a_{i}$ al menos en el $i$-ésimo decimal, o sea $b_{i} \neq a_{i i}$. Por lo tanto, $b$ no es igual a ningún número de la serie $a_{1}, a_{2}, \ldots, a_{i}, \ldots$, luego la hipótesis de que esta serie contenía a todos los números reales en el intervalo $(0,1)$ es falsa, ya que existe al menos un número real $b$ que no está en este conjunto. Luego, el conjunto de los números reales en el intervalo $(0,1)$, y por ende, el conjunto de los números reales, no puede ser puesto en correspondencia biyectiva con el conjunto de los números naturales (Jech, 2011).

Estas propiedades de los números infinitos eran tan desconcertantes que muchos filósofos y matemáticos de la época se resistieron a aceptar la teoría creada por Cantor. Incluso, él mismo, admitía que ciertas conclusiones que se derivaban de su trabajo eran extrañas y contrarias a la intuición (Arrigo y D’Amore, 1999). Notemos que, para él, pensar en términos del infinito actual imponía aceptar que una secuencia infinita tenía un estado final resultante en 
el infinito como, por ejemplo, cuando un círculo es concebido como un polígono regular con un número infinito de lados.

Además, la idea del infinito actual era controvertida, porque a menudo llevaba a contradicciones. Incluso los matemáticos que aceptaban su existencia, comprendían las dificultades que surgían en consecuencia (Dauben, 1990). Un ejemplo clásico de esto es la "afirmación" $k / 0=\infty$, donde $k$ es una constante finita. Esta "afirmación" se basa en la idea de que, a medida que el denominador se vuelve progresivamente más pequeño el valor de la fracción aumenta indefinidamente. O sea, si en el infinito el denominador se hace 0 , entonces el valor de la fracción se hace $\infty$ (mayor que cualquier valor finito). El problema es que aceptar este resultado también significaría aceptar que $0 . \infty=k$, es decir, que la multiplicación de cero por infinito podría ser igual a cualquier número $k$ finito.

Una de las paradojas más cautivadoras sobre el infinito, que aparecieron precisamente en esta época: la de Hilbert y su hotel infinito, planteaba algunas de estas ideas contradictorias. Por ejemplo, el resultado de multiplicar infinito por un número finito sigue siendo infinito, pero si multiplicamos infinito por un infinitesimal lo que se obtiene es indeterminado, o sea, $k . \infty=$ $\infty$, si $k$ es finito, pero si $k$ es un infinitesimal, entonces no se conoce el resultado.

Los trabajos de Cantor hicieron posible la incorporación de instrumentos conceptuales y técnicas de manipulación del infinito que abrieron camino a nuevas disciplinas matemáticas. Definió dos tipos de números infinitos refiriéndose a dos nociones del infinito actual distintas: los ordinales transfinitos, como una extensión de la noción de número ordinal al caso infinito, y los cardinales transfinitos, como extensión de la noción de conteo al caso infinito. De hecho, usando el cardinal del conjunto potencia de un conjunto, demostró que había un conjunto infinito y ordenado de cardinales transfinitos. Con su teoría sobre los números transfinitos, logró proporcionar a las matemáticas una estructura que integraba diferentes infinitos. Sin embargo, rechazó los infinitesimales porque parecían contradecir la concepción misma del infinito actual y carecían de estructura propia como cuerpo teórico matemático. Posteriormente, en el siglo XX, Robinson (1966), basado en el trabajo de Skolem (1934) demostraría lo contrario, creando el análisis no estándar, introduciendo el infinito no-estándar y usando la teoría de modelos en la fundamentación de su trabajo. Su enfoque axiomático y riguroso, le permitió introducir los 
infinitesimales en su sentido actual, como números hiperreales no nulos, cuyo valor absoluto es menor que cualquier número real (Bell, 2008).

\section{Aspectos y Metáforas presentes en los diferentes períodos históricos}

Analizaremos ahora las ideas y paradojas contra-intuitivas que surgieron a lo largo de la historia en relación al infinito matemático, desde la perspectiva de la $E C$ y la lingüística cognitiva. Esto, a su vez, nos permitirá entender los procesos de abstracción que se construyen a partir de estas estructuras conceptuales contradictorias, y las razones por las cuales estos resultados nos parecen contraintuitivos. El análisis también nos podría mostrar por qué la naturaleza del infinito actual no puede entenderse en términos de verdades trascendentales (o platónicas), o en términos de la lógica formal. Entre los mecanismos que usaremos están los sistemas de aspectos (procesos iterativos y continuos, estructuras perfectivas e imperfectivas, con estados iniciales, estados resultantes, etc.), y las metáforas e integraciones conceptuales.

El estudio del aspecto nos permite comprender, por ejemplo, la estructura cognitiva de acciones iterativas como "respirar" y de acciones continuas como "moverse", a medida que se manifiestan a través del lenguaje en las situaciones cotidianas. El aspecto nos informa acerca de la estructura de acciones que tienen puntos de inicio y final inherentes, como por ejemplo, "saltar"; acerca de acciones que solo tienen puntos de partida como por ejemplo, "salir"; o acerca de acciones que solo tienen puntos finales, como por ejemplo, "llegar". Cuando las acciones tienen puntos finales, también tienen estados resultantes. Por ejemplo, "llegar" (cuya estructura como aspecto tiene punto final), implica que una vez finalizada la acción, se está ubicado en el lugar de llegada. Cuando una acción no tiene punto final, no tiene estado resultante. Muchas dimensiones de la estructura de eventos se pueden estudiar a través del aspecto, que tiene dos distinciones: el aspecto perfectivo, que tiene finalización inherente y el aspecto imperfectivo, que no tiene finalización inherente. Por ejemplo, la estructura prototípica de "saltar" tiene finalización inherente cuando el sujeto que realiza la acción toca el suelo o alguna otra superficie, por lo que "saltar" tiene un aspecto perfectivo. "Volar", por el contrario, no tiene finalización inherente, la acción prototípica de "volar" en sí misma no implica ningún final específico. Cuando el sujeto que "vuela" toca el suelo, el acto mismo de tocar el suelo pone fin a la acción, pero no pertenece a "volar" en sí, por lo que "volar" tiene un aspecto imperfectivo. Un proceso con aspecto imperfectivo puede ser conceptualizado como un proceso continuo 
iterativo (con puntos finales intermedios y resultados intermedios). La capacidad cognitiva humana de concebir algo continuo en términos iterativos resulta ser muy importante cuando se trata del infinito. De la misma manera, un proceso continuo sin fin se puede conceptualizar como un proceso iterativo infinito (Lakoff y Núñez, 2000).

Desde el punto de vista del aspecto, el infinito potencial implica la consideración de procesos que pueden o no tener un punto de partida, pero que niegan explícitamente la posibilidad de tener un punto final, o sea, no tienen finalización, ni estado final resultante. Luego, los procesos involucrados en el infinito potencial tienen aspecto imperfectivo.

Ahora notemos que las caracterizaciones de conjuntos infinitos dadas a lo largo de la historia, desde Galileo hasta Cantor, estudiaban las nociones cotidianas de "misma cantidad que" y "más que", que se basan, de manera natural, en nuestra experiencia con colecciones finitas. Cuando consideramos que el conjunto A (finito) tiene la "misma cantidad" de elementos que el conjunto B (finito), esto significa que si para cada elemento de A, quitamos un elemento correspondiente de B, entonces ningún elemento de B queda "sobrante".

Si extendemos la idea de "sobrante" a conjuntos infinitos, y nos acercamos a la pregunta: ¿hay más números naturales que números pares?, podemos hacer coincidir los elementos de ambos conjuntos y llegar a la conclusión de que hay más números naturales que números pares, porque en el conjunto de los números naturales quedan números impares "sobrantes". Sin embargo estos dos conjuntos son emparejables en el sentido de que podemos poner en correspondencia biyectiva sus elementos. Por lo tanto, el "emparejamiento" y la "misma cantidad" son dos ideas que tienen la misma extensión para colecciones finitas, pero que son cognitivamente diferentes y tienen estructuras inferenciales que difieren de manera importante. Ya Galileo en siglo XVII había comprendido esto.

En sus investigaciones sobre los conjuntos infinitos, Dedekind y Cantor utilizaron el concepto de "emparejamiento" en lugar de nuestro concepto cotidiano de "misma cantidad". Al hacerlo, y al abandonar implícitamente la idea de "sobrante", establecieron una metáfora conceptual, en la que el concepto "misma cantidad" se conceptualiza en términos del otro, es decir, en términos de “emparejamiento". En la Figura 2 se muestra el mapeo de esta metáfora conceptual "misma cantidad como emparejamiento", que le permitió a Cantor crear todo el 
aparato conceptual de su teoría, y le da un significado metafórico preciso a la comparación del número de elementos o cardinalidad de conjuntos infinitos (Núñez, 2005).

Figura 2. Metáfora conceptual Misma cantidad como emparejamiento.

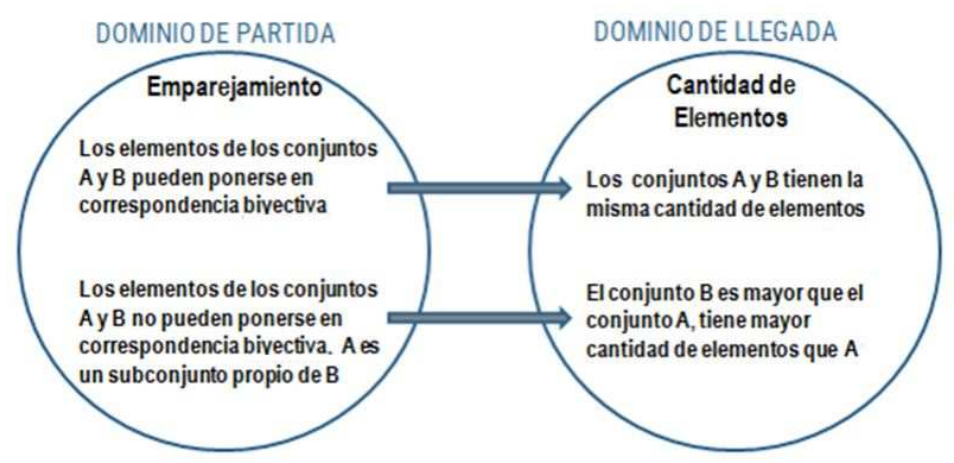

Fuente: Elaboración propia

Es importante notar que esta nueva concepción de "cantidad" establecida por Dedekind y Cantor es de naturaleza metafórica, y que además de establecer el "emparejamiento", para poder extender la noción de cardinalidad a conjuntos infinitos, se necesita ignorar activamente la cláusula "sobrante" implícita en la noción ordinaria de "más que” (Núñez, 2005).

En relación con el infinito actual, Lakoff y Núñez (2000) proponen que su concepción se basa en la llamada metáfora básica del infinito $(M B I)$, mediante la cual, los procesos que continúan indefinidamente se conceptualizan de manera que alcanzan un estado final, una especie de "completamiento metafórico". Además, en este trabajo se plantea que la $M B I$ es el único mecanismo conceptual humano que se encarga de la creación de todo tipo de infinito actual en matemáticas, desde puntos infinitos en el plano, hasta sumas infinitas, conjuntos infinitos y números y límites infinitesimales. La $M B I$ es una integración conceptual, que constituye una asociación o enlace entre dominios conceptuales diferentes. Específicamente en este caso se parte de dos dominios que se conectan para "proyectarse" selectivamente en un dominio de llegada donde se desarrolla una estructura nueva. El primer dominio de entrada es un espacio que implica procesos iterativos completados (con aspecto perfectivo). El segundo dominio de entrada implica procesos iterativos sin fin (con aspecto imperfectivo), y por lo tanto caracteriza procesos relacionados con el infinito potencial. En el dominio integrado de llegada emerge una estructura que se infiere para caracterizar los procesos involucrados en el infinito 
actual. En la Figura 3 se muestra las correspondencias entre los dominios de entrada y las proyecciones hacia el espacio integrado que constituye el dominio de llegada.

Figura 3. La metáfora básica del infinito (MBI)

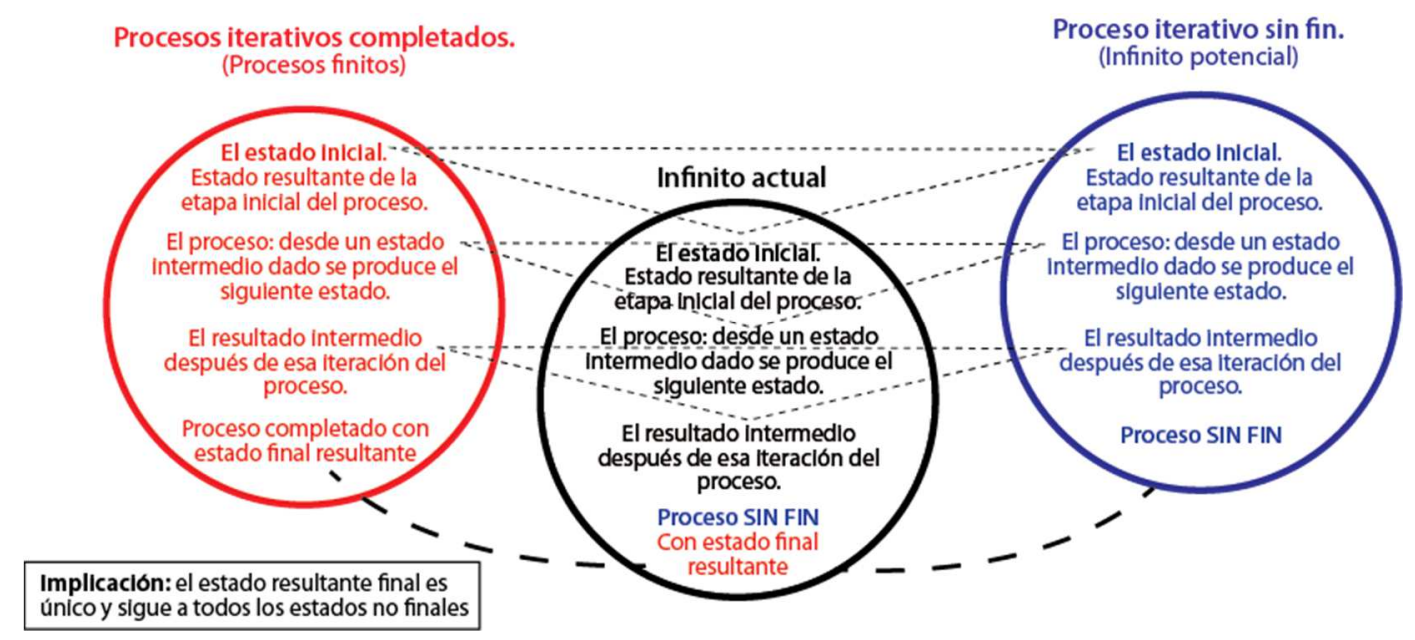

Fuente: Núñez (2005, p.1730) (Traducción propia)

Es importante ver que la riqueza y peculiaridad de la $M B I$ es su organización y estructura como mezcla de doble alcance (Fauconnier y Turner, 2002). La correspondencia entre los dos espacios de entrada implica a todos los elementos con la excepción del último, el único elemento que distingue esencialmente un proceso finito de un proceso potencialmente infinito. Esto indica un conflicto entre una caracterización de un proceso que explícitamente tiene fin y estado resultante final, y una caracterización que explícitamente representa un proceso interminable, sin estado final resultante. A menudo estos conflictos no se resuelven, no se forma ningún espacio integrado a partir de los dominios originales de entrada.

Un ejemplo de esto nos lo proporciona Galileo en siglo XVII, cuando al observar que los números naturales y los números cuadrados perfectos podían ser puestos en correspondencia biyectiva, no pudo llegar a ninguna conclusión que hubiera requerido completar el proceso sin fin. Si el conjunto de los números cuadrados es un subconjunto de los números naturales, entonces forman una colección más pequeña que los números naturales. Frente a esta situación paradójica, Galileo concluyó que no debía compararse conjuntos cuando uno o ambos tenían una cantidad infinita de elementos. Claramente ni Galileo ni los matemáticos de su época disponían de la definición de conjunto infinito dada por Dedekind dos siglos después, que concebía la posibilidad de hacer coincidir los elementos de un conjunto infinito con uno de sus 
subconjuntos propios, ni podía considerar que esta era una propiedad esencial de los conjuntos infinitos y no una extraña patología.

Como Lakoff y Núñez (2000) han señalado, una implicación crucial de la $M B I$ es que el estado final resultante es único y sigue a cada estado no final. La singularidad proviene de los dominios de entrada, donde para cualquier proceso completado, el estado final resultante es único. Además, el hecho de que el estado resultante sea final, significa que no hay estado final anterior. Del mismo modo, no hay un estado final posterior del proceso. Es decir, no hay otro estado del proceso que resulte de la finalización del proceso y siga el estado final. Luego, los cardinales transfinitos son el resultado de una combinación magistral de la metáfora conceptual "misma cantidad como emparejamiento" y de la integración conceptual MBI realizada por Cantor.

Por último veremos que aunque Robinson construyó su infinitesimal actual de otra manera, también hizo uso implícito de la $M B I$ para darle "completamiento metafórico" al infinitesimal de Leibniz, tomando el conjunto de los números reales mayores que 0 y menores que $1 / n$ (con $n$ entero positivo); de tal manera que éstos, junto con sus inversos aditivos y multiplicativos y los correspondientes elementos identidad y neutro, satisfacieran los nueve primeros axiomas de los números reales (excluyendo la propiedad Arquimideana). Luego construyó una sucesión de tales conjuntos de números reales en los intervalos $(0,1),(0,1 / 2),(0$, $1 / 3), \ldots,(0,1 / n), \ldots$. La Figura 4 muestra este proceso de construcción iterativo infinito, que a través de la $M B I$ llega a su estado final, obteniéndose así el conjunto de infinitesimales en el sentido actual que constituyen las creaciones metafóricas de la $M B I$ en este caso.

Figura 4. Completamiento metafórico del Infinitesimal

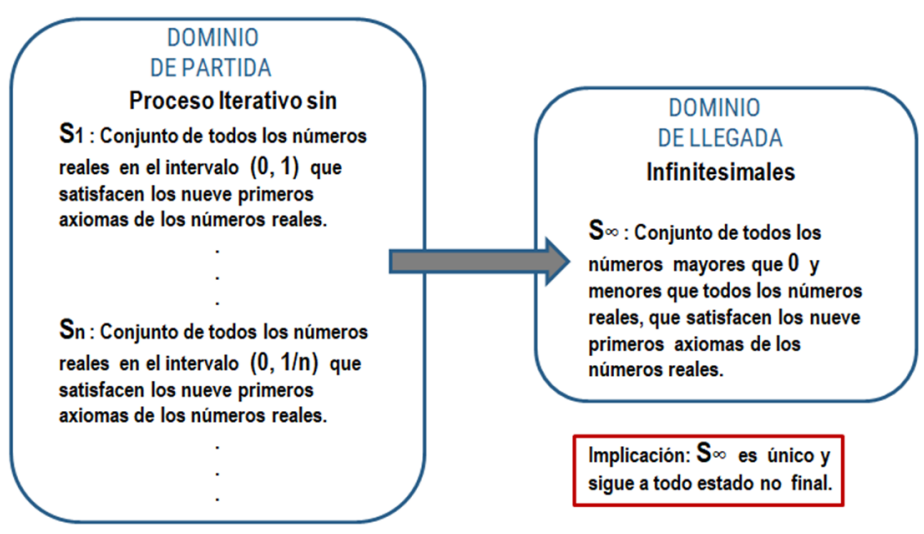

Fuente: Elaboración propia 
Luego, los infinitesimales de Robinson, así como los cardinales y los ordinales transfinitos de Cantor, "se completaron metafóricamente" a través de la $M B I$.

\section{Conclusiones}

En este artículo abordamos el análisis histórico del infinito matemático bajo el lente de la metáfora conceptual desde una perspectiva epistemólogica, tratando de precisar obstáculos que impidieron, por largos períodos de nuestra historia la aceptación del infinito actual, permitiéndose solamente la existencia del infinito potencial. Mostramos cómo el desarrollo de ciertas metáforas conceptuales condujo a un proceso de axiomatización del infinito actual, que culminó con los trabajos de Georg Cantor y Abraham Robinson.

Sus trabajos marcaron un hito y el final del largo recorrido realizado por el infinito matemático a lo largo de la historia, desde su existencia en sentido potencial hasta su existencia en sentido actual. Este proceso iniciado en la antigua Grecia, pasó por cuatro estadíos fundamentales, tal como se mostró anteriormente en nuestro análisis. Además, nuestro estudio también mostró que todas las instancias del infinito actual "se completaron metafóricamente" a lo largo de la historia a través de la $M B I$. Como se muestra en la Figura 5, mientras que en las matemáticas que se desarrollaron en diferentes épocas, los infinitos actuales se caracterizaron por diferentes conjuntos de axiomas en campos aparentemente no relacionados, cognitivamente, se pueden caracterizar por este único mecanismo cognitivo.

Figura 5. Momentos históricos y el desarrollo de la $\mathrm{MBI}$

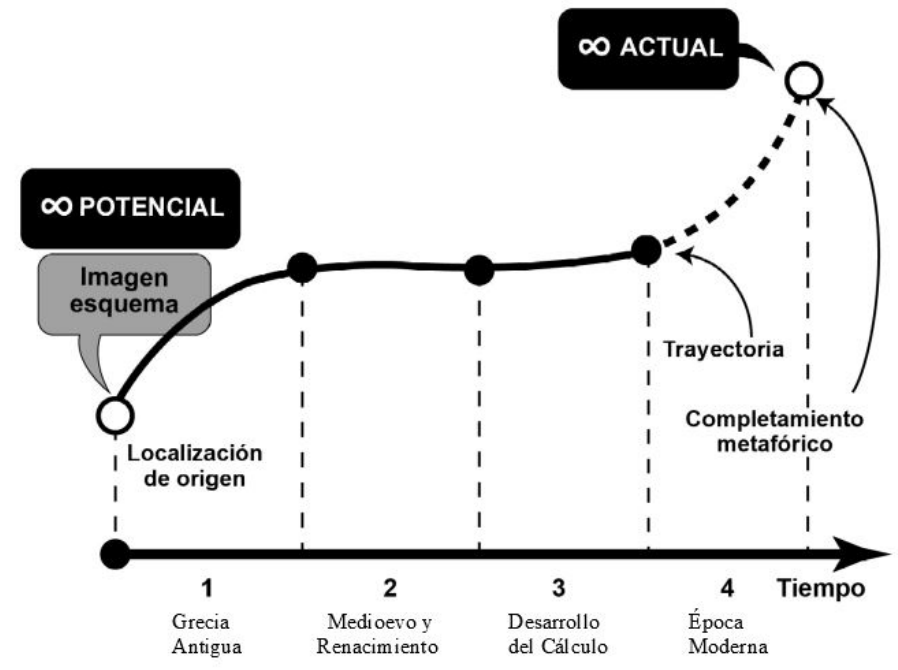

Fuente: Elaboración propia 
Los cardinales y ordinales transfinitos fueron el resultado de una combinación magistral de la metáfora conceptual "misma cantidad como emparejamiento" y de la integración conceptual MBI. Estas ideas y los mecanismos cognitivos subyacentes involucrados en los trabajos de Dedekind y Cantor, se pueden estudiar bajo el lente de la $E C$ y de la lingüística cognitiva, que se apoya en el estudio de estructuras conceptuales humanas, del lenguaje y de peculiaridades de nuestro cerebro (como la percepción visual y la experiencia espacial), y proporciona un análisis plausible de la naturaleza y del devenir histórico del infinito matemático en sus categorías principales, el infinito potencial y el infinito actual.

La ingeniosa extensión metafórica realizada por Dedekind y Cantor del concepto de "emparejamiento" y su aplicación a conjuntos infinitos constituyó un extraordinario logro conceptual en las matemáticas. En el proceso, no solo crearon un nuevo concepto matemático, sino también, nuevas matemáticas, que no podrían haber sido creadas sólo con nuestras nociones ordinarias cotidianas de "misma cantidad que" y "más que". Algo muy similar ocurrió con los infinitesimales de Robinson en el siglo XX. Desafortunadamente, inicialmente muchos matemáticos ignoraron la naturaleza metafórica de los nuevos infinitos creados por ellos, en parte porque les resultaron contra-intuitivos. En la actualidad, sus teorías han sido ampliamente aceptadas, y paradójicamente, a menudo se concluye que hay algo incorrecto en nuestra intuición cuando se trata del infinito. Nuestro análisis cognitivo muestra que no hay nada de malo con nuestra intuición "per se". En nuestro sistema conceptual ordinario, estas teorías no pueden ser ciertas. No porque nuestra intuición esté equivocada, o porque nuestro lenguaje cotidiano sea impreciso, sino porque es una inferencia hecha dentro de una estructura conceptual diferente, con una estructura inferencial diferente. De acuerdo con nuestra noción ordinaria de "más que", de hecho hay más números naturales que positivos, o cuadrados, así como hay más enteros que números naturales (Núñez, 2005). Hay una lógica cognitivamente estructurada muy precisa que subyace a esta inferencia, y que podemos hacer tan rigurosa como queramos. La falta de rigor, entonces, no es el problema. Si además consideramos que nuestros mecanismos cognitivos se ven limitados por nuestras sensaciones motosensoriales, que en el caso de los números transfinitos se basan en esquemas de contenedor para colecciones finitas y sus jerarquías, en mecanismos básicos de discriminación cuantitativa determinados genéticamente, y en experiencias cinestésicas relacionadas con la comparación de tamaños y correspondencia 
de elementos (Lakoff y Núñez, 2000), entonces podemos comprender el origen de nuestras intuiciones y hacernos conscientes de estas limitaciones.

De todo el análisis anterior concluimos que la reconstrucción histórico-epistemológica del infinito matemático bajo la mirada de la metáfora conceptual pudiera ser de gran utilidad para la comprensión de dificultades a las que se enfrentan estudiantes y profesores en los procesos de enseñanza y aprendizaje relacionados con el infinito matemático, y debería ser un elemento a considerar en las agendas de investigación dentro de la didáctica de las matemáticas. Este es un concepto fundamental desde el punto de vista epistemológico, y el análisis histórico de la formación de disciplinas matemáticas a lo largo de la historia, nos muestra que es imposible estudiarlo aisladamente y sin referencia a otros conceptos matemáticos de alta relevancia en la educación superior.

Por otra parte, desde nuestra perspectiva este tipo de estudios es especialmente importante, no solo porque incide notablemente en la formación matemática de profesores y estudiantes, contribuyendo a ampliar y enriquecer el horizonte cultural desde donde se aborda el estudio de los conceptos matemáticos, sino también porque nos permite identificar metáforas y mecanismos cognitivos que el profesor debe considerar y el estudiante debe desarrollar para superar dificultades y obstáculos, y así lograr una comprensión adecuada de un determinado concepto matemático, y en este caso en particular, del infinito matemático.

\section{Agradecimientos}

Este trabajo ha sido desarrollado como parte de la Tesis Doctoral en Educación Matemática de Tamara Díaz-Chang y está parcialmente financiado por la Dirección de Investigación de la Vicerrectoría de Investigación y Postgrado de la Universidad de Los Lagos, D.U. 430.

\section{Referencias}

Aristóteles. (trad. 1985). The complete Works of Aristotle. The revised Oxford translation. In J. Barnes (Ed.). New Jersey, United States: Princeton University Press.

Arquímedes. (trad. 1897). The Sand Reckoner of Archimedes. New York: Cambridge University Press.

Arrigo, G. y D'Amore, B. (1999). Lo veo, pero no lo creo: obstáculos epistemológicos y didácticos para la comprensión del infinito actual. Educación Matemática, 11(01), 5-24. 
Arrigo, G. y D'Amore, B. (2004). Otros hallazgos sobre los obstáculos en la comprensión de algunos teoremas de Georg Cantor. Educación Matemática, 16 (2), 5-20.

Bagni, G.T. (2004). Exhaustion argument and limit concept in the History of Mathematics: educational reflections. In Furinghetti, F, Kaiser, S. \& Vretblad, A. (Eds.), Proceedings of HPM-2004, History and Pedagogy of Mathematics, Uppsala, 94-103.

Bagni, G.T. (2006). History of Calculus from Eudoxus to Cauchy. Historical investigation and interpretation and mathematics education. In Furinghetti, F. Kaiser, S. \& Tzanakis, C. (Eds.), Proceedings of HPM-2004 \& ESU-4, Revised edition (pp. 529-536). Crete: Emedia, University of Crete.

Bell, E. T. (2003). The Development of Mathematics. Dover Publications, New York.

Bell, J. L. (2008). A primer of infinitesimal analysis. New York: Cambridge University Press.

Bolzano, B. (1991). Las paradojas del infinito. D.F., México: Universidad Nacional Autónoma de México.

Bussotti, P., Tapp, C. (2009). The influence of Spinoza's concept of infinity on Cantor's set theory. Studies in History and Philosophy of Science, 40, 25-35.

Cobb, P., Yockel, E. \& Wood, T. (1992). A constructivist alternative to the representational view of the mind in mathematics education. Journal for Research in Mathematics Education, 23(1), 2-33.

Comrie, B. (1976). Aspect. Cambridge Textbooks in Linguistics.

Dauben, J. W., (1990). Georg Cantor: His Mathematics and Philosophy of the Infinite. Princeton University Press, Princeton, NJ.

Durán, A. (2003). Euler y los infinitos (grandes y pequeños). Recuperado el 2 de mayo 2020 de http://upcommons.upc.edu/e-prints/bitstream/2117/1985/1/ mblanco_paper_icehve.pdf

Eves H., Carroll V. (1966). An introduction to the foundations and fundamental concepts of matemathics, New York: Holt, Rinehart and Winston.

Fauconnier, G. \& Turner, M. (2002). The way we think. New York: Basic Books.

Fischbein, E. (2001). Tacit models and infinity. Educational studies in Mathematics, 48, 309329.

Flusberg, P., Matlock, T. \& Thibodeau, S. (2019). The role of metaphor in communication and thought. Language and Linguistics Compass, 13(5).

Font, V. (2007). Epistemología y Didáctica de las Matemáticas. En F. Ugarte (ed.) Reportes de investigación. n. 21, serie C, II Coloquio Internacional sobre la Enseñanza de las Matemáticas (pp. 1-48). Lima, Perú: PUCP

Galilei, G. (trad.1954). Dialogues concerning two new sciences, Dover Publications, New York.

Gallese, V. \& Lakoff, G. (2005). The brain's concepts: The role of the sensory-motor system in conceptual knowledge. Cognitive Neuropsychology, 22 (3), 455-479.

Guthrie, W. K. C. (2000). A History of Greek Philosophy. Cambridge University Press.

Jech, T. (2011). Set Theory, Stanford Encyclopedia of Philosophy.

Lakoff, G. \& Núñez, R. (2000). Where Mathematics comes from. New York: Basic Books.

Lucrecio. (trad. 1985). De la naturaleza de las cosas. Barcelona, España: Orbis. 
Noblit \& Hare (1998) Metha-etnography: synthesizing qualitative studies. Sage Publications, California.

Núñez, R. (2005) Creating mathematical infinities: Metaphor, blending, and the beauty of transfinite cardinals. Journal of Pragmatics, 37, 1717-1741.

O’ Connor, J. J., \& Robertson, E. F. (2002). Mac Tutor History of Mathematics. Recuperado el 2 de Mayo de 2010 de http://www. history.mcs.stand.ac.uk/HistTopics/Infinity.html

Paéz, T. D. (2013) Las matemáticas a lo largo de la historia. Madrid, España: Visión Libros.

Robinson, A. (1966). Non-standard Analysis. Princeton University Press.

Rohrer, T. (2006). Image Schemata in the Brain. En From Perception to Meaning: Image Schemas in Cognitive Linguistics, Beate Hampe and Joe Grady, eds., Berlin: Mouton de Gruyter. 165-196.

Rosch, E., Thompson, E. y Varela, F. (1991). The embodied mind: Cognitive science and human experience. MIT Press, Cambridge, MA.

Sanhueza, D. (2015). Consideraciones sobre el infinito en la filosofía de Kant. Revista de Filosofía, 71, 151-162.

Sfard, A. (2008). On two metaphors for learning and the dangers of choosing just one. Educational Researcher, 27(2), 4-13.

Skolem, T. (1934). Über die Nicht-charakterisierbarkeit der Zahlenreihe mittels endlich oder abzählbar unendlich vieler Aussagen mit ausschließlich Zahlenvariablen, Fundamenta Mathematicae. 23 (1), 150-161.

Stillwell, J. (2010). Mathematics and its history. Springer Science+Business Media. New York, USA.

Tall, D. (1991). The Psychology of Advanced Mathematical Thinking. En Tall, D. (Ed.) Advanced Mathematical Thinking, Kluwer: Hollad, 3-21.Wilson \& Gibbs (2007).

Autoras

Tamara Díaz Chang. Candidata a Doctora en Educación Matemática por el Departamento de Ciencias Exactas de la Universidad de Los Lagos, Osorno, Chile. Profesora del Instituto de Ciencias Físicas y Matemáticas e Investigadora del Centro Interdisciplinario de Estudios del Sistema Nervioso (CISNe) de la Universidad Austral de Chile. Campus Isla Teja, Valdivia, Chile. E-mail: tamara.diaz@uach.cl.

Elizabeth Hernández Arredondo. Doctora en Ciencias, especialidad Matemática Educativa por el Centro de Investigación y de Estudios Avanzados del IPN, Cinvestav-IPN México. Profesora de la Universidad de Los Lagos, Osorno, Chile. Dirección postal: Postgrado en Educación Matemática, Departamento de Ciencias Exactas, Calle Lord Cochrane 1039, Osorno, Chile. E-mail: elizabeth.hernandez@ulagos.c1 